\title{
A Device Improves Signs and Symptoms of TMD
}

\author{
Annalisa Monaco $\mathbb{D}^{1},{ }^{1}$ Davide Pietropaoli $\mathbb{D},{ }^{1}$ IAPNOR, ${ }^{2}$ Barry C. Cooper, ${ }^{3}$ \\ and Eleonora Ortu $(\mathbb{D})^{1}$ \\ ${ }^{1}$ University of L'Aquila, Department of Life, Health and Environmental Sciences, San Salvatore Hospital, \\ Building Delta 6-Unit of Dentistry, V.le San Salvatore, L'Aquila 67100, Italy \\ ${ }^{2}$ I.A.P.N.O.R. International Academy of Posture and Neuromyofascial Occlusion Research, Viale Gino Moretti 37, \\ 63074 San Benedetto del Tronto (AP), Italy \\ ${ }^{3}$ Division of Translational Oral Biology, School of Dental Medicine, State University of New York, Stony Brook, New York, \\ NY 14214, USA
}

Correspondence should be addressed to Eleonora Ortu; eleortu@gmail.com

Received 19 November 2018; Revised 13 March 2019; Accepted 7 April 2019; Published 6 May 2019

Academic Editor: Marina De Tommaso

Copyright $\odot 2019$ Annalisa Monaco et al. This is an open access article distributed under the Creative Commons Attribution License, which permits unrestricted use, distribution, and reproduction in any medium, provided the original work is properly cited.

\begin{abstract}
Background. Temporomandibular joint dysfunction (TMD) therapy remains an open challenge for modern dentistry. Herein, we propose a new neuromuscular lingual device able to reduce signs and symptoms of TMD in female patients with chronic orofacial pain. Methods. 50 females with myofascial TMD according to RDC/TMD were randomly assigned to study $(n=25)$ and control groups $(n=25)$. At T0, both groups received sEMG/KNG and pain evaluation by the VAS scale. The study group received the ELIBA device (lingual elevator by Balercia) constructed under ULF-TENS (ultra-low-frequency transcoutaneous electrical nervous stimulation). Subjects were instructed to use ELIBA at least for $16 \mathrm{~h} / \mathrm{day}$. After 6 months (T1), both groups underwent to sEMG/KNG and VAS revaluation. Results. T1 study group compared to controls showed a significant reduction in total $(p<0.0001)$ and mean $(p<0.0001)$ sEMG values, as well as a significant increase in both maximum vertical mouth opening ( $p=0.003)$ and maximum velocity in mouth opening $(p=0.003)$ and closing $(p<0.0001)$. Interestingly, a significant reduction in pain measured by VAS $(p<0.0001)$ was reported. Conclusions. After 6 months, the ELIBA device is able to significantly reduce TMD-associated myogenous pain and to promote the enhancement of sEMG/KNG values. Practical Implications. ELIBA can be considered as a new device, potentially useful for head-neck pain relief in patients suffering from chronic TMD. In addition, its use promotes a muscles relaxation inducing freeway space increase. This characteristic makes it particularly useful for rehabilitation of patients with not enough space for construction of conventional orthotics or neuromuscular bites.
\end{abstract}

\section{Introduction}

Neuromuscular dentistry employs ultra-low-frequency transcutaneous electrical neural stimulation (ULF-TENS) to obtain a reduction of muscular tone utilizing surface electromyography (sEMG) to evaluate physiological freeway space [1-4]. Decrease of muscles' electrical resting hyperactivity and increase of interocclusal distance after ULFTENS are necessary conditions to permit the fabrication of oral cavity devices, such as neuromuscular orthotics, which conform to the neuromuscular philosophy concepts and practices. Comfortable outcome for patients receiving this therapy has been documented [5]. In a small percentage of clinical cases (10-15\%), it is not possible to get a reduction in electromyography resting electrical activity values and, above all, an increase of freeway space after ULF-TENS. This peculiar condition does not permit the use of neuromuscular orthotics to rehabilitate those patients [6]. One of the causes of failure to achieve reduction of electromyography values after ULF-TENS for some patients might be the lack of relaxation of suprahyoid muscles (digastric, stylohyoid, geniohyoid, and mylohyoid) and cervical muscles, which are agonists for mandibular and respiratory function [7]. In traditional orthodontic therapy, several devices have been 
proposed to improve tongue posture and function with the goal of modulating resting tongue position [8-11]. Modification of tongue posture may entail an alteration of the neck and lower jaw posture in the rest position [12]. Among these devices, the lingual elevator (ELIBA) is the only one which employs ULF-TENS for its construction and permits the adaptation of the sublingual space (oral floor/suprahyoid muscles) specific to the anatomy and neuromuscular physiology of each individual. This procedure will be described in Methods [12-14]. The purpose of our research is to evaluate the effect of ELIBA (lingual elevator by Balercia) in patients suffering from temporomandibular disorders (TMDs) who did not experience either a significant reduction in electromyography resting values or an increase of interocclusal distance following ULF-TENS. A secondary goal of our work is to assess, with an individual scale, the positive effects on patient subjective symptoms after several months of therapy.

\section{Materials and Methods}

2.1. Subjects. This study was conducted in accordance with the Declaration of Helsinki. The Committee on Ethics in Science of the University of L'Aquila, L'Aquila, Italy, approved the study, and written informed consent was obtained from each subject and electronically stored as suggested by our institutional guidelines. This study was registered at: NCT02946645.

Fifty Caucasian patients (mean age 36.8; SD 8.5) who fulfilled the following criteria were included in the study group: (1) female gender; (2) age less than 50 years; (3) craniocervical myogenous TMD; (4) pain duration longer than 3 months; (5) reduction of freeway space and impairment sEMG activity after TENS according to Konchak et al. [6]; (6) presence of complete permanent dentition, with the possible exception of the third molars.

Patients were excluded from the study if they met one or more of the following criteria: (1) presence of systemic or metabolic diseases; (2) eye diseases or visual defects; (3) history of local or general trauma; (4) neurological or psychiatric disorders; (5) muscular diseases; (6) bruxism, as diagnosed by the presence of parafunctional facets and/or anamnesis of parafunctional tooth clenching and/or grinding; (7) pregnancy; (8) assumed use of antiinflammatory, analgesic, antidepressant, opioid, or muscle relaxant medications; (9) smoking; (10) fixed or removable prostheses; (11) fixed restorations that affected the occlusal surfaces; and (12) either previous or concurrent orthodontic or orthognathic treatment [3].

The diagnosis of myofascial-type TMD was provided after clinical examination by a trained clinician according to group 1a and $1 \mathrm{~b}$ of the Research Diagnostic Criteria for TMD (RDC/TMD), in a blinded manner (RC) $[15,16]$.

The enrolled subjects were randomly assigned to one of two groups: control group $(n=25)$ and study group $(n=25)$. The two groups were checked for age (study 36.41, SD 6.41; control 37.02, SD 9.15). Measurement of actual pain was recorded for each subject in a visual analog scale (VAS) of pain [17]. Each subject underwent sEMG and jaw tracking
(KNG or computerize mandibular scan) recordings (TIME 0) according to the Monaco protocol in a blind fashion [7]. At the end of recordings, the study group underwent sublingual myoprint (sapphire ${ }^{\circledR}$ H Bosworth, Scokie, IL, USA) registration for the construction of the ELIBA device under TENS stimulation, while the control group did not. The study group received the device, and they were educated to use it at least sixteen hours a day. Checkups were carried out every 15 days for a total of six months. After six months, both control and study groups received a second sEMG/KNG (TIME 1) in a blind manner.

2.2. sEMG/Jaw Tracking Recording Procedure. Briefly, all examinations were performed using an 8-channel surface electromyograph with simultaneous acquisition, common grounding to all channels, and filters of $50 \mathrm{~Hz}$. Data obtained were displayed and stored on an electromyography device (K7/EMG ${ }^{\circledR}$, Myotronics-Noromed, Inc., Tukwila WA, USA), with disposable electrodes (Duotrode ${ }^{\circledR}$, bipolar surface electrodes $\mathrm{Ag}-\mathrm{AgCl}, 20 \mathrm{~mm}$ center to center distance, Myotronics-Noromed, Inc., Tukwila WA, USA), for sEMG recording. Resting electrical activity in the right masseter (RMM), left masseter (LMM), right anterior temporalis (RTA), left anterior temporalis (LTA), right digastric (RDA), left digastric (LDA), right sternocleidomastoid (RSCM), and left sternocleidomastoid (LSCM) muscles were recorded. The sEMG recordings and muscle activity was expressed as the root mean square (RMS) of the amplitude, expressed in $\mu \mathrm{v}$ [18]. Jaw tracking (Kinesiographic KNG) recordings were performed using a kinesiograph (K7/CMS ${ }^{\circledR}$; MyotronicsNoromed, Inc., Tukwila, WA, USA) that measures jaw movements in three dimensions with an accuracy of $0.1 \mathrm{~mm}$. A lightweight array weighing 113 grams containing 8 magnetic sensors affixed to the bridge of the nose and held in place with Velcro straps at the back of the skull tracked the motion of a $0.1 \mathrm{oz}$ magnet (CMS Magnet; MyotronicsNoromed, Inc., Tukwila WA, USA) that was attached to the labial gingiva beneath the mandibular incisor teeth in the mandibular midpoint with an adhesive gel. The kinesiography and electromyography were interfaced with a computer for data storage and subsequent software analysis (K7 Program, Myotronics-Noromed, Inc., Tukwila WA, USA).

Electrodes were positioned on LMM, RMM, LTA, and RTA, as previously described [19], as well on RDA, LDA [20], and LSC and RSC [21, 22]. A template was used to permit the exact repositioning of the electrodes on repeated testing sessions. The ground electrode, which was larger than the others and ensured a very good contact with the skin, was positioned on the subject's forehead to ensure a common reference to the differential input of the amplifier. The kinesiographic array was mounted on the bridge of the nose and aligned for the optimal position of the magnet for the recording of kinematic movements which was monitored by software. Inherent electromyographic noise was tested through K7 software for each channel and a value of $0.0 \mu \mathrm{v}$ could be accepted. In case of excess of noise, a new electrode was placed in an appropriate location for that muscle.

sEMG/KNG recordings include the following: 
(i) Scan 9-sEMG activity at basal condition with eyes lightly closed

(ii) Scan 1-maximal voluntary mandibular opening

(iii) Scan 2-velocity of jaw movements during mandibular opening and closing

(iv) Scan 3-freeway space recording at the incisor point

(v) Motor trigeminal TENS stimulation

(vi) Scan 10-sEMG activity at basal condition with closed eyes after TENS

(vii) Scan 4-freeway space recording after TENS

Note: sEMG or KNG scans with artefacts due to swallowing or aberrant head or mandibular movements were discarded and the recordings were performed again.

2.3. TENS Stimulation Procedure. The method for sensory TENS was described previously [23-27]. Briefly, a J5 Myomonitor ${ }^{\circledR}$ TENS Unit device (Myotronics-Noromed, Inc., Tukwila, WA, USA) with disposable electrodes (Myotrode SG Electrodes ${ }^{\circledR}$, Myotronics-Noromed, Inc., Tukwila, WA, USA) was used. This device generates a repetitive synchronous and bilateral stimulus delivered at 1.5second intervals, with adjustable amplitude of approximately $0-24 \mathrm{~mA}$, a duration of $500 \mu \mathrm{s}$, and a frequency of $0.66 \mathrm{~Hz}$. The two TENS electrodes were placed bilaterally anterior to the tragus of each ear to provide neural stimulation of the mandibular division of the trigeminal nerve (CM V div 2). The electrode placement position was located between the coronoid and condylar processes of the mandible and was identified by manual palpation of the zone anterior to the tragus; a third common electrode was placed in the center of the back of the neck [3]; two landmarks were made on the patient's chin and nose, which were used to find the mandibular skull dimension with a compass or caliper, after sensory stimulation with the ULF-TENS. The amplitude of TENS stimulation started at $0 \mathrm{~mA}$, with the stimulator device turned on and the rheostat, which controls the amplitude, positioned at 0 . The amplitude of stimulation was progressively increased at a rate of $0.6 \mathrm{~mA} / \mathrm{s}$ until the patients reported the sensation of pricking at the electrodes level. Once the sensory threshold has been set, the stimulus was administered with the ULF-TENS lasting 4/6 minutes, remembering to check the position between the arches before the use of the resin and subjects undergoing the test were asked to relax. Resin was prepared (Keystone Bosworth Sapphire ${ }^{\circledR}$ Blue/Clear, Myerstown, PA, USA) in the powder/ liquid ratio 2.75 (12 grams of powder)/8 cc liquid.

2.4. ELIBA Construction. The acronym ELIBA ${ }^{\circledR}$ stands for Balercia lingual elevator. Professor Luigi Balercia, with the help of the research members of IAPNOR, were the first to build and use this device for rehabilitation purposes, in order to stimulate and support the maintenance of the posture of the tongue in all patients affected by swallowing deviated during speech therapy, orthognathic therapy and as an aid for dysphagia and Down syndrome (Figure 1). The device is designed as an oral device that can be firmly anchored in the lower jaw (Figure 1). It is inserted into a triangular portion of the sublingual space, bounded anteriorly and laterally by the mandible and by the lingual surfaces of the teeth, inferiorly from the oral floor (mainly constituted by the mylohyoid muscle) and superiorly by the ventral surface of the tongue (Figure 1). In order to take an impression of sublingual space, a previous stimulation with ULF-TENS is used. The patient is asked to rest the apex of the tongue against the physiological point (retroincisal papilla) and keep the tongue relaxed in this position for the duration of the procedure. After 4/6 minutes of sensory ULF-TENS, the patient had to open her mouth to allow the introduction, with a suitable syringe, of the impression material (Sapphire Resin, Myoprint), according to the reports liquid powder as already described, of consistency plastic, so as to completely fill the sublingual space. The subject was asked to close her mouth in the previously described position, keeping the tongue relaxed with the apex against the physiological point (retroincisal papilla). Initial hardening of the impression resin was expected up to a rubbery resilient state. When the resin reached a solid but elastic consistency, before complete hardening, it was extracted from the oral cavity and inserted into the master model to complete the polymerization (Figure 1) [28]. The clinician indicated to the laboratory the distal lateral limits of the ELIBA $^{\circledR}$ device, which served for the realization of the artifact and the most suitable retentive devices for the stabilization of $\mathrm{ELIBA}^{\circledR}$. The following procedures were carried out by technicians qualified to manufacture the ELIBA ${ }^{\circledR}$ product, following standardized duplication procedures. In the construction of ELIBA ${ }^{\circledR}$ the device manufacturer (dental laboratory) must follow precise clinical indications on the lateral distal borders, highlighted by the clinician on the impression taken by the patient with the Sapphire resin. The finishing process carried out by the dental laboratory must strictly respect the indications of the clinician (Figure 1), paying close attention to the intimate contact with the lingual surfaces of the teeth of the lower arch (Figure 1). The realization of the retentive systems of the product ELIBA ${ }^{\circledR}$, hooks, bands or other, are exclusively prescribed by the clinician. Only the passivation of the product and the retention of the device are the responsibility of the dental laboratory. The ELIBA ${ }^{\circledR}$ product must be stable and not have occlusal contacts [12, 13, 29-32].

\section{Statistical Analysis}

The statistical analysis was conducted using STATA $10^{\circledR}$ (StataCorp LP, College Station, TX, USA). Normal distribution of data was tested by the Shapiro-Wilk test. In order to compare within group the EMG-KIN and VAS data, the $t$ test for paired data was performed. The comparison between groups was carried out with $t$-test for unpaired data.

For EMG-mean parameter was calculated the algebraic mean of the sum of the mean rms of each muscle recorder according to Cooper [5]. The mean rms was automatically calculated by K7I program at the end of 15-second period of EMG recording. 


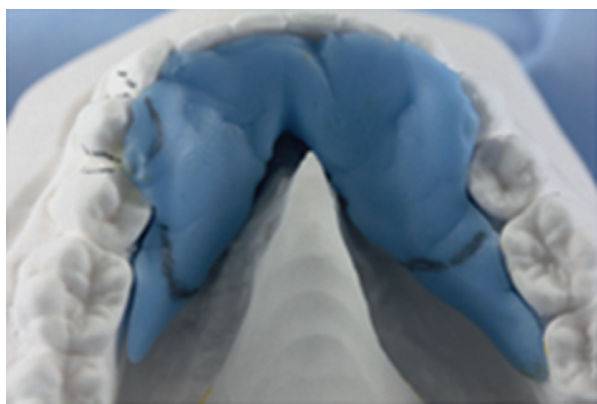

(a)

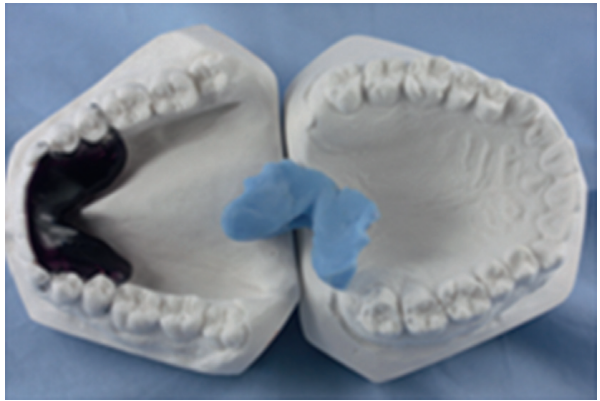

(c)

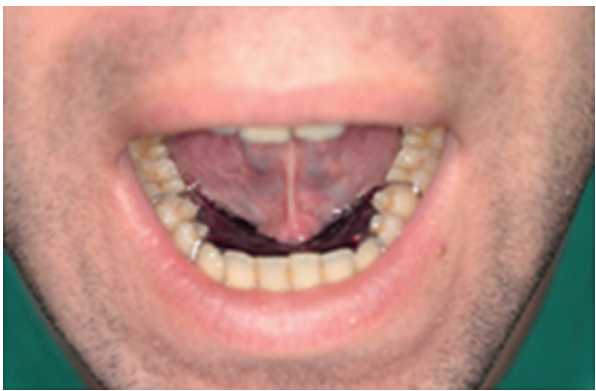

(e)

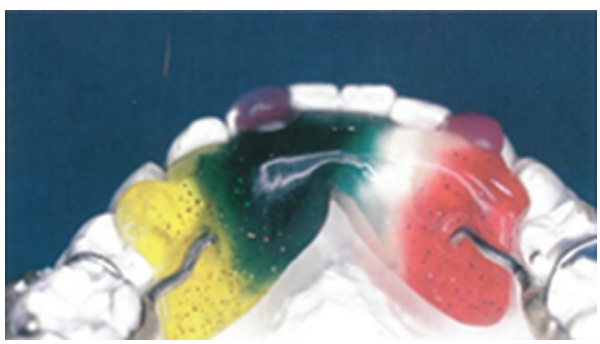

(g)

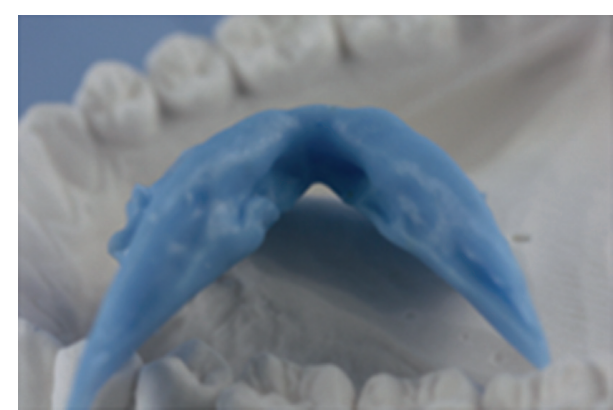

(b)

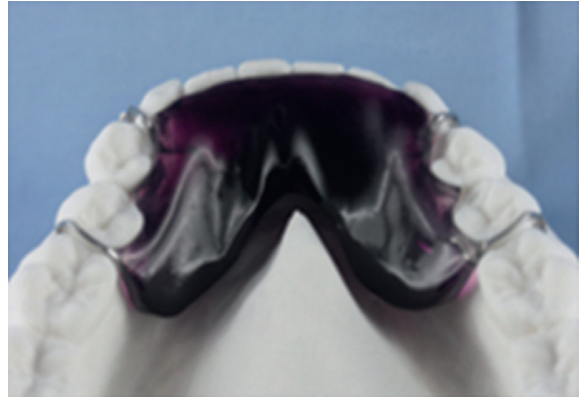

(d)

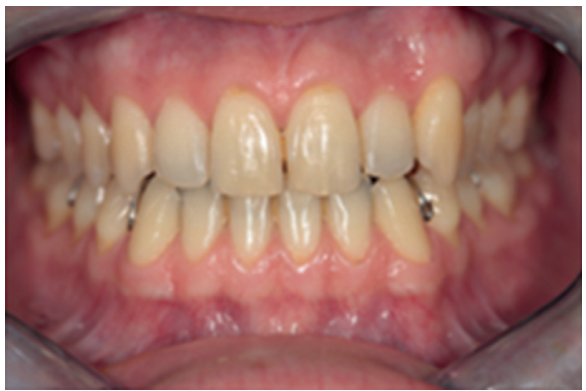

(f)

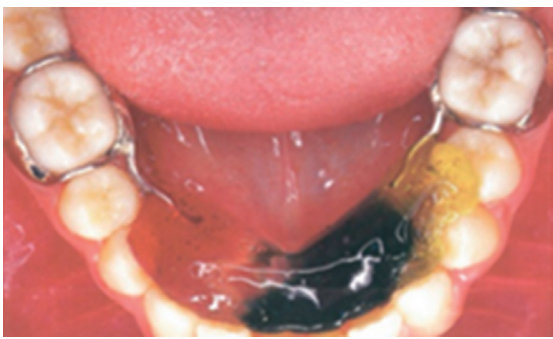

(h)

Figure 1: ELIBA construction phases and oral aspect of the device. (a) Marking of the lateral/distal borders of the ELIBA ${ }^{\circledR}$ device, as specified by the clinician. (b) Detail of the caudal part of the acrylic impression. (c) The ELIBA ${ }^{\circledR}$ appliance on the plastic cast and impression of the ELIBA ${ }^{\circledR}$ device, as finished in the dental laboratory following the instructions of the clinician. (d) Enlargement of the device ELIBA ${ }^{\circledR}$ applied on the plastic cast with hooks in front area in cobalt chrome. (e-h) Intraoral views of the ELIBA appliance fitted to the oral cavity of the patient. (f) The ELIBA ${ }^{\circledR}$ device without occlusal contacts. (g) ELIBA ${ }^{\circledR}$ on plastic cast with connection arm hook joined with weld.

For maximum opening (MO) of the mouth, maximum velocity of opening (MVO), and maximum velocity of closing (MVC), the mean of three consecutive movement cycles for each parameter was chosen.

Our hypothesis was that base data (TIME 0) did not differ significantly between the control and ELIBA groups, whereas the comparison of the two groups after therapeutic intervention at TIME 1 could differ if ELIBA, if the beneficial effects of the appliance use were documented with EMG, KNG and VAS data obtained.

The level of significance was set at $p<0.05$ for all tests. The results are expressed in terms of mean and standard deviation (SD), while in the bar plots, mean and standard error (SE) were represented. 


\section{Results}

Tables 1 and 2 report the EMG/KNG comparability of the two groups in the base condition (TIME 0) according to EMG-KNG inclusion criteria.

Table 1 shows the EMG data of ELIBA and control groups in the base condition (TIME 0). No statistical significant reduction of values has been seen comparing before and after ULF-TENS in both groups (within-group comparison); for an immediate comparison, at a glance, see the value of EMG-mean: neither ELIBA (2.42 vs 2.16) nor control (2.13 vs 2.39) showed significant difference. No significance has been seen in EMG statistics comparing (between-group statistics) the two groups in the base condition (TIME 0).

The KNG measures of mandible velocity of opening and closure and the maximum opening of the mandible are plotted in Table 3. In TIME 0 , no differences have been seen in the two groups. No difference between groups in VAS was noted (Tables 3 and 4). The EMG-KNG and VAS data in TIME 0 allowed the comparability of the two groups.

Table 2 refers to the KNG data and comparison of the freeway space (FWS) after TENS in TIME 0 and TIME 1. According to inclusion criteria, the mean values of vertical dimension (Vert.) of the FWS after ULF-TENS were lower than $1.5 \mathrm{~mm}$ in both groups. No significant differences were found between the two groups. In TIME 1, the FWS Vert. after ULF-TENS of the ELIBA group increases growing beyond the inclusion value of $1.5 \mathrm{~mm}$. (0.92 vs 1.55 ; $p=0.011)$. The control group does not increase the FWS vert. in TIME 1 comparing TIME 0 . The between-group comparison indicates significant differences in vert. and lat. in TIME 1 (1.55 vs 0.78: $p=0.001$ and 0.39 vs 0.54 ; $p=0.028$, respectively).

Table 5 reports the EMG data recorded before ULFTENS in TIME 0 and at the TIME 1 condition comparing the two groups. The data show that the ELIBA group demonstrates a reduction in the overall electrical activity in the rest condition (EMG-mean 2.42 vs $1.91 ; p=0.031$ ). This significance results by the sum of the reduction of the single muscles that individually are not able to reach the significance. The control group increases the overall rest EMG activity in TIME 1 (EMG-mean 2.13 vs $2.63 ; p=0.017$ ) because all muscles show higher resting electrical activity values in TIME 1 compared to TIME 0: LTA, RMM, LDA, and RDA increase reaches the significance in TIME 1 in the control group. The between-group comparison shows in TIME 0 no statistical significance in all muscles and EMGmean, as already listed in Table 1. In TIME 1, all muscles and EMG-mean values are significantly lower in the ELIBA group compared to the control group.

Next, we compared the sEMG data before and after ULFTENS within and between groups. As indicated in Table 5, the comparison between groups before ULF-TENS shows significantly lower resting electrical activity value in all muscles in the ELIBA patient group. After ULF-TENS, the data show that the most significant data are the decrease of EMG in the ELIBA group (1.67 vs $2.44 ; p<0.001)$. The RTA, LDA, RDA, and RSC reached the level of significance between the two groups, lower in the ELIBA group than in the control.

Table 3 shows the KNG and VAS comparison between TIME 0 and TIME 1 between and within the groups. The ELIBA group increases in a significant manner all the KNG (maximum opening, maximum velocity of opening, and maximum velocity of closing) measures in TIME 1 compared to TIME 0 . The VAS decreases significantly in this group in TIME 1 ( 7.13 vs $2.07 ; p \leq 0.001$ ). The control group does not show differences in all KNG and VAS data comparing TIME 0 and TIME 1 . The between-group comparison in TIME 1 indicates a significant difference in KNG and VAS measures, higher KNG and lower VAS values in ELIBA comparing control group.

\section{Discussion}

Data that were obtained from this study suggest that the use of ELIBA appliances in patients suffering from TMD

(1) significantly reduces resting electrical activity seen in sEMG values

(2) significantly increases both maximum vertical mouth opening and maximum velocity in mouth opening and closing values

(3) significantly increases the vertical component of the FWS after ULF-TENS

(4) significantly reduces pain

This oral device, initially born for orthodontic purposes, can help patients suffering from TMJ to relax the stomatognathic system (EMG and kinesiographic values). It can also help the patient in the reduction of the pain [12]. Usually electromyography values decrease and freeway space increases after ULF-TENS. When this phenomenon does not occur, in a few TMD patients, the clinician is faced with a big challenge in developing a diagnosis and a correct treatment planning in accordance with the neuromuscular gnathology philosophy and practices. However, assessing data of each research group, it was found that with this additional therapeutic aid, electromyography values tend to decrease after ULF-TENS [5, 33-35].

Konchak in 1988 showed that in some patients, neither electromyography values decreased nor did interocclusal distance increase after ULF-TENS [6]. A few patients, 10\%, did not relax neither chewing nor postural muscles after ULF-TENS. In $5 \%$ of subjects, freeway space even reduces. These patients cannot be treated pursuant to classic neuromuscular philosophy because ULF-TENS did not achieve muscle relaxation permitting the mandible to assume a physiologic mandible rest position. Moreover, lack of freeway space after ULF-TENS (less than one millimeter) does not permit the fabrication of neuromuscular orthotics that, even if very small, would further obliterate freeway space preventing the physiologic relaxation of muscles. Some authors have proposed the reduction of dental anatomy using burs in these TMD patients who do not respond favorably to ULF-TENS. However, unless patients already wear prosthetics, this solution is definitely 
TABLE 1: EMG values of ELIBA and control groups in the base condition (TIME 0) before and after ULF-TENS (S9 before ULF-TENS; S10 after ULF-TENS).

ELIBA TIME $0 \quad$ Control TIME 0
Statistics between the groups

Before TENS After TENS

\begin{tabular}{|c|c|c|c|c|c|c|c|c|}
\hline S9 (before TENS) & S10 (after TENS) & $p$ & S9 (before TENS) & S10 (after TENS) & $p$ & $p$ & $p$ & \\
\hline LTA & $2.98(1.55)$ & $1.73(0.72)$ & 0.014 & $2.17(1.18)$ & $2.00(0.67)$ & 0.576 & 0.121 & 0.290 \\
\hline LMM & $1.68(0.78)$ & $1.05(0.35)$ & 0.005 & $1.69(0.91)$ & $1.23(0.36)$ & 0.117 & 0.966 & 0.161 \\
\hline RMM & $1.65(0.68)$ & $1.67(0.92)$ & 0.930 & $1.38(0.65)$ & $1.71(0.93)$ & 0.335 & 0.283 & 0.907 \\
\hline RTA & $2.55(1.66)$ & $2.19(1.67)$ & 0.393 & $2.05(0.93)$ & $2.96(1.72)$ & 0.055 & 0.313 & 0.226 \\
\hline LSC & $3.08(2.88)$ & $3.38(3.40)$ & 0.768 & $2.93(2.26)$ & $3.19(1.30)$ & 0.688 & 0.878 & 0.839 \\
\hline LDA & $2.07(0.79)$ & $2.43(1.40)$ & 0.212 & $1.87(0.71)$ & $2.34(0.79)$ & 0.125 & 0.487 & 0.825 \\
\hline $\mathrm{RDA}$ & $2.19(0.63)$ & $2.05(0.66)$ & 0.519 & $2.10(1.06)$ & $2.33(0.65)$ & 0.427 & 0.787 & 0.253 \\
\hline RSC & $3.18(2.34)$ & $2.82(1.74)$ & 0.522 & $2.83(1.45)$ & $3.38(1.43)$ & 0.307 & 0.630 & 0.344 \\
\hline sEMG-mean & $2.42(0.71)$ & $2.16(0.75)$ & 0.272 & $2.13(0.62)$ & $2.39(0.26)$ & 0.104 & 0.238 & 0.283 \\
\hline
\end{tabular}

Statistics: $t$-test for paired data for within-group comparison and for unpaired data in between-group comparison. For abbreviations, refer to Materials and Methods.

TABLE 2: Kinesiographic measurements and comparison of FWS (freeway space) after ULF-TENS in ELIBA and control groups in TIME 0 and TIME 1.

\begin{tabular}{|c|c|c|c|c|c|c|c|c|c|}
\hline & \multicolumn{3}{|c|}{ ELIBA group } & \multicolumn{4}{|c|}{ Control group } & \multicolumn{2}{|c|}{$\begin{array}{l}\text { Statistics between } \\
\text { the groups }\end{array}$} \\
\hline & TIME 0 & TIME 1 & $p$ & TIME 0 & TIME 0 & TIME 1 & $p$ & $\begin{array}{c}\text { TIME } 0 \\
p\end{array}$ & $\begin{array}{c}\text { TIME } 1 \\
p\end{array}$ \\
\hline \multirow{3}{*}{ FWS after TENS } & Vert.. & $0.92(0.47)$ & $1.55(0.73)$ & 0,011 & $0.88(0.28)$ & $0.78(0.15)$ & 0,246 & 0.78 & 0.001 \\
\hline & $\mathrm{AP}$ & $0.65(0.32)$ & $0.99(0.66)$ & 0,092 & $0.76(0.27)$ & $0.75(0.30)$ & 0,900 & 0.31 & 0.220 \\
\hline & Lat. & $0.41(0.13)$ & $0.39(0.21)$ & 0,919 & $0.41(0.92)$ & $0.54(0.13)$ & 0,004 & 0.74 & 0.028 \\
\hline
\end{tabular}

Statistics: $t$-test for paired and unpaired data.

TABLE 3: KNG and VAS data and comparison between and within groups at TIME 0 and TIME 1.

\begin{tabular}{|c|c|c|c|c|c|c|c|c|}
\hline & \multicolumn{3}{|c|}{ ELIBA group } & \multicolumn{3}{|c|}{ Control group } & \multicolumn{2}{|c|}{$\begin{array}{l}\text { Statistics between the } \\
\text { groups }\end{array}$} \\
\hline & TIME 0 & TIME 1 & $p$ & TIME 0 & TIME 1 & $p$ & $\begin{array}{c}\text { TIME } 0 \\
p\end{array}$ & $\begin{array}{c}\text { TIME } 1 \\
p\end{array}$ \\
\hline $\mathrm{MO}$ & $293.13(44.26)$ & $344.53(35.95)$ & 0.002 & $291.2(35.26)$ & $303.53(31.51)$ & 0.321 & 0.896 & 0.003 \\
\hline MVO & $231.67(64.32)$ & $324.07(98.51)$ & 0.006 & $266.33(55.83)$ & $228.27(55.54)$ & 0.072 & 0.126 & 0.003 \\
\hline MVC & $249.2(57.48)$ & $317.2(37.44)$ & 0.001 & $236.47(44.14)$ & $207.33(35.07)$ & 0.056 & 0.502 & $\leq 0.001$ \\
\hline VAS & $7.13(1.06)$ & $2.07(1.22)$ & $\leq 0.001$ & $6.80(0.94)$ & $7.13(0.92)$ & 0.334 & 0.370 & $\leq 0.001$ \\
\hline
\end{tabular}

The EMG/KNG data used to support the findings of this study have not been made available because they are private data. Bold text represents statistical difference $(p<0.05)$. MO = maximum opening; $\mathrm{MVO}=$ maximum velocity of opening; $\mathrm{MVC}=$ maximum velocity of closing.

TABLE 4: sEMG values of ELIBA and control groups in TIME 1 before and after ULF-TENS.

\begin{tabular}{|c|c|c|c|c|c|c|c|c|}
\hline & \multicolumn{3}{|c|}{ ELIBA TIME 1} & \multicolumn{3}{|c|}{ Control TIME 1} & \multicolumn{2}{|c|}{$\begin{array}{l}\text { Statistics between the } \\
\text { groups }\end{array}$} \\
\hline & S9 (before TENS) & S10 (after TENS) & $p$ & S9 (before TENS) & S10 (after TENS) & $p$ & $\begin{array}{c}\text { Before TENS } \\
p\end{array}$ & $\begin{array}{c}\text { After TENS } \\
p\end{array}$ \\
\hline LTA & $2.28(0.99)$ & $1.79(0.85)$ & 0.043 & $2.91(0.61)$ & $2.37(0.71)$ & 0.009 & 0.049 & 0.053 \\
\hline LMM & $1.37(0.39)$ & $1.26(0.57)$ & 0.313 & $2.06(0.74)$ & $1.29(0.27)$ & 0.003 & 0.004 & 0.841 \\
\hline RMM & $1.85(1.09)$ & $1.42(0.66)$ & 0.008 & $2.59(0.68)$ & $1.81(0.64)$ & 0.005 & 0.034 & 0.108 \\
\hline RTA & $1.83(0.49)$ & $1.49(0.65)$ & 0.004 & $2.46(0.94)$ & $2.48(1.52)$ & 0.969 & 0.031 & 0.032 \\
\hline LSC & $1.61(0.48)$ & $2.5(1.75)$ & 0.070 & $2.86(1.25)$ & $1.81(0.91)$ & 0.033 & 0.002 & 0.191 \\
\hline LDA & $1.99(0.66)$ & $1.4(0.57)$ & $<0.0001$ & $2.55(0.79)$ & $3.49(1.32)$ & 0.023 & 0.042 & $\leq 0.001$ \\
\hline $\mathrm{RDA}$ & $2.41(0.33)$ & $1.58(0.72)$ & $<0.0001$ & $2.81(0.56)$ & $2.29(0.59)$ & 0.027 & 0.025 & 0.006 \\
\hline RSC & $1.97(0.89)$ & $1.88(1.17)$ & 0.534 & $2.79(1.22)$ & $3.67(1.42)$ & 0.101 & 0.045 & 0.001 \\
\hline EMG-mean & $1.91(0.49)$ & $1.67(0.39)$ & 0.0001 & $2.63(0.44)$ & $2.4(0.41)$ & 0.263 & $\leq 0.001$ & $\leq 0.001$ \\
\hline
\end{tabular}

Statistics: $t$-test for paired data in within-group comparison and for unpaired data in between-group comparison. For abbreviations, refer to Materials and Methods. Bold text represents statistical difference $(p<0.05)$. 
TABLE 5: Comparisons of TIME 0 vs TIME 1 of EMG values before ULF-TENS.

\begin{tabular}{|c|c|c|c|c|c|c|c|c|}
\hline & \multicolumn{3}{|c|}{ ELIBA S9 (before TENS) } & \multicolumn{3}{|c|}{ Control S9 (before TENS) } & \multicolumn{2}{|c|}{$\begin{array}{l}\text { Statistics between the } \\
\text { groups }\end{array}$} \\
\hline & TIME 0 & TIME 1 & $p$ & TIME 0 & TIME 1 & $p$ & $\begin{array}{c}\text { TIME } 0 \\
p\end{array}$ & $\begin{array}{c}\text { TIME } 1 \\
p\end{array}$ \\
\hline$\overline{\text { LTA }}$ & $2.98(1.55)$ & $2.28(0.99)$ & 0.154 & $2.17(1.18)$ & $2.91(0.61)$ & 0.045 & 0.121 & 0.049 \\
\hline LMM & $1.68(0.78)$ & $1.37(0.39)$ & 0.178 & $1.69(0.91)$ & $2.06(0.74)$ & 0.236 & 0.966 & 0.004 \\
\hline RMM & $1.65(0.68)$ & $1.85(1.09)$ & 0.552 & $1.38(0.65)$ & $2.59(0.68)$ & $\leq \mathbf{0 . 0 0 1}$ & 0.283 & 0.034 \\
\hline RTA & $2.55(1.66)$ & $1.83(0.49)$ & 0.123 & $2.05(0.93)$ & $2.46(0.94)$ & 0.237 & 0.313 & 0.031 \\
\hline LSC & $3.08(2.88)$ & $1.61(0.48)$ & 0.070 & $2.93(2.26)$ & $2.86(1.25)$ & 0.913 & 0.878 & 0.002 \\
\hline LDA & $2.07(0.79)$ & $1.99(0.66)$ & 0.766 & $1.87(0.71)$ & $2.55(0.79)$ & 0.020 & 0.487 & 0.042 \\
\hline $\mathrm{RDA}$ & $2.19(0.63)$ & $2.41(0.33)$ & 0.229 & $2.10(1.06)$ & $2.81(0.56)$ & 0.031 & 0.787 & 0.025 \\
\hline RSC & $3.18(2.34)$ & $1.97(0.89)$ & 0.076 & $2.83(1.45)$ & $2.79(1.22)$ & 0.925 & 0.630 & 0.045 \\
\hline EMG-mean & $2.42(0.71)$ & $1.91(0.49)$ & 0.031 & $2.13(0.62)$ & $2.63(0.44)$ & 0.017 & 0.238 & $\leq 0.001$ \\
\hline
\end{tabular}

Statistics: $t$-test for paired data in within-group comparison and for unpaired data in between-group comparison. For abbreviations, refer to Materials and Methods. Bold text represents statistical difference $(p<0.05)$.

discouraged, because it is not reversible and it does not assure a clinical beneficial outcome $[36,37]$. The lower jaw rest position and consequent interocclusal freeway space can be partly influenced both by the tongue position between dental arches and by head and neck posture, mostly occurring through reciprocal interaction in essential functions such as breathing and swallowing.

The postural relationship between the hyoid, mandible, and neck is still controversial; Valenzuela et al. even state that there is no postural correlation [38]. Castro suggests a relation between sEMG activity of omohyoid muscle, which arises from the upper border of the scapula and inserts into the lower border of the body of the hyoid bone and anterior belly of digastric muscle during tongue movements and changes of position of the apex of the tongue [20]. Others found that the hyoid bone position generally had strong linear correlations with the positions of the head, jaw, and cervical vertebrae C1-C2 [39]. The cervical area between C1 and $\mathrm{C} 3$ receives proprioceptive afferent fibers from suboccipital, sternocleidomastoid, and trapezius muscles [40]. On the other hand, in healthy people, there is correlation between sEMG activity of sternocleidomastoid muscles and posture of the head and neck during physiological acts as swallowing and maximum voluntary clenching. This relationship tends to worsen in TMD patients [41].

In addition, as definitely demonstrated by Fitzgerald [42], the proprioception of the extrinsic and intrinsic muscles of the tongue, which are innervated with motor fibers by hypoglossal nerve (CN XII), is supplied by $\mathrm{C} 1$ and $\mathrm{C} 2$ coming from the hypoglossal ansa sharing with the above cited muscles of neck. It seems probable that this correlation, that has anatomical and functional reasons, could have some clinical effect. For example, the position of hyoid, where extrinsic muscles of tongue are inserted, is correlated to tongue posture and to craniocervical angle: they both appear abnormal in sleep apnea patients in contrast to healthy subjects. Sleep apnea obstructive syndrome involves the tongue, lower jaw, pharynx, and neck in a pathophysiological way [43]. In sleep apnea patients, the mandibular position is abnormal, in part because during sleep, the tongue is more retruded than in healthy people $[44,45]$.
Functionally, the mandible, neck/head, and tongue are strictly associated in some reflex activities which induce a perpetual arrangement of reciprocal muscular tone [46, 47]. Stimulation of the lingual nerve, sensorial secondary branch of mandibular division of the trigeminal nerve (CN Vdiv3), determines the discharge of hypoglossal nerve fibers which concurrently cause tongue retrusion [48]. At the same time, opening the mouth determines the enhancement of sEMG activity of the genioglossus muscle and mandible and tongue posture can reciprocally influence [49]. For example, sensorial stimulation with light pressure stimulus of temporal muscle induces activation of motor neurons of hypoglossal nucleus, demonstrating a strict relationship between the postural muscles of the mandible and tongue [50]. The existence of an anatomical and functional relationship between muscles of neck and tongue has been experimentally documented by Edwards et al. [40]. In the intermediate nucleus of the medulla, the proprioception of suboccipital muscles, sternocleidomastoid, and trapezius is monosynaptical and related with the nucleus of the twelfth cranial nerves and with the nucleus of the solitary tract, this justifying the functional union among neck, head, and tongue and the vegetative answers inducted by postural variations of every element of this circuit. Among them are also some afferent fibers to the intermediate nucleus coming from vestibular and oculomotor nucleus [47]. It is also interesting to note that the tongue posture, position of apex of the tongue, and freeway space are correlated to watchfulness and mood. In fact, during watching emotional videos, the tension will be higher, the tongue posture will be lower, and interocclusal distance will be smaller [51]. The posture of apex of the tongue is related to other anatomical regions, specially the head and neck, and significantly influences orientation reactions in difficult visual search tasks [52].

The results of our work generally seem to be in accordance with concepts previously expressed. sEMG values at rest show a significant reduction of electrical potentials in muscles directly related with the lower jaw position (anterior temporalis and masseter muscle) and neck (sternocleidomastoid) making supposition that the ELIBA device action may partly be due both to sensorial stimulus to the lingual 
nerve and postural, determined by variation of position of tongue and apex of the tongue (C1-C2). This action may induce a different relation among all the parts related to the same system and involved in this phenomenon (head, neck, mandible, and tongue). Increase of kinesiography values of maximum mouth opening and maximum velocity in mouth opening can be interpreted as an enhancing in neuromuscular and sensorial balance [53].

In our work, we did not mathematically assess the position of tongue, apex of the tongue, and head and neck region, so we do not have data about spatial modifications of the anatomical regions involved in ELIBA. Our scientific assessment is limited to sEMG and kinesiography, so it is not excluded that more equilibrated muscles, and TMJ values can be obtained in the same spatial position of the head, neck, lower jaw, and tongue. However, even if data about it are not available, it is probable that an ELIBA appliance introduced in the sublingual space induces spatial variation of tongue position. About this assertion, the literature claims that introducing orthodontic devices in spaces useful for tongue determines modification of posture and functional movements of the tongue [33]. Employment of oral splints (orthoses) for TMD therapy still remains controversial because there is not a universal consensus as to diagnosis and pathogenesis of TMD [54-56]. Published studies assessing neuromuscular and not neuromuscular oral splints [5] have shown improvement of sEMG parameters and subjective symptoms. In our study, habitual occlusion has not been modified, and ELIBA use in fact does not require alteration of preexistent occlusion. The purpose of our study was to demonstrate that it is possible to employ an oral device being able to modify tongue posture and ameliorate both symptoms and improve sEMG and KNG values in TMD patients whose sEMG and KNG parameters do not improve after ULFTENS, and these patients cannot be included in a traditional neuromuscular gnathology protocol. It is relevant to specify that our results are not related with an occlusal modification such as occlusal adjustment according with neuromuscular or other gnathology philosophies. A limitation of our work is that it has not been effectuated a comparison with a control placebo group. We cannot assess how much of the positive clinical outcome may be due to placebo effect. A future paper will compare ELIBA clinical results with the outcome obtained with a neuromuscular occlusal splint and with placebo therapy $[2,3,5,57]$.

\section{Data Availability}

The EMG/KNG data used to support the findings of this study have not been made available because they are private data.

\section{Conflicts of Interest}

IAPNOR holds a registered patent on the ELIBA ${ }^{\circledR}$ device, c/o the Italian Ministry of Economic Development, patent office.

\section{Acknowledgments}

This article was written in agreement and courtesy of the IAPNOR association, as the ELIBA ${ }^{\circledR}$ device is a registered patent c/o the Italian Ministry of Economic Development, patent office.

\section{References}

[1] B. Jankelson, "Three-dimensional orthodontic diagnosis and treatment. A neuromuscular approach," Journal of Clinical Orthodontics: JCO, vol. 18, no. 9, pp. 627-36, 1984.

[2] B. C. Cooper, "More about TMD and SEMG," Journal of the American Dental Association, vol. 137, no. 10, pp. 1362-1363, 2006.

[3] A. Monaco, R. Cattaneo, L. Mesin, E. Ortu, M. Giannoni, and D. Pietropaoli, "Dysregulation of the descending pain system in temporomandibular disorders revealed by low-frequency sensory transcutaneous electrical nerve stimulation: a pupillometric study," PloS One, vol. 10, no. 4, Article ID e0122826, 2015.

[4] S. Tecco, S. Mummolo, E. Marchetti et al., "sEMG activity of masticatory, neck, and trunk muscles during the treatment of scoliosis with functional braces. A longitudinal controlled study," Journal of Electromyography and Kinesiology, vol. 21, no. 6, pp. 885-892, 2011.

[5] B. C. Cooper and I. Kleinberg, "Establishment of a temporomandibular physiological state with neuromuscular orthosis treatment affects reduction of TMD symptoms in 313 patients," $C R A N I O^{\circledR}$, vol. 26, no. 2, pp. 104-117, 2008.

[6] P. A. Konchak, N. R. Thomas, D. T. Lanigan, and R. M. Devon, "Freeway space measurement using mandibular kinesiograph and EMG before and after TENS," Angle Orthodontist, vol. 58, no. 4, pp. 343-50, 1988.

[7] A. Monaco and R. Cattaneo, Elettromiografia e kinesiografia, Per la Clinica Odontoiatrica: Principi di Odontoiatria Neuro Mio Fasciale, Libreria Cortina S.R.L., Milan, Italy, 2007.

[8] R. Condò, M. Costacurta, C. Perugia, and R. Docimo, "A typical deglutition: diagnosis and interceptive treatment: a clinical study," European Journal of Paediatric Dentistry: Official Journal of European Academy of Paediatric Dentistry, vol. 13, no. 3, pp. 209-14, 2012.

[9] D. Ciavarella, L. Lo Russo, M. Mastrovincenzo et al., "Cephalometric evaluation of tongue position and airway remodelling in children treated with swallowing occlusal contact intercept appliance (S.O.C.I.A.)," International Journal of Pediatric Otorhinolaryngology, vol. 78, no. 11, pp. 1857-1860, 2014.

[10] E. Ortu, D. Pietropaoli, M. Ortu, M. Giannoni, and A. Monaco, "Evaluation of cervical posture following rapid maxillary expansion: a review of literature," The Open Dentistry Journal, vol. 8, no. 1, pp. 20-27, 2014.

[11] E. Ortu, M. Giannoni, M. Ortu, R. Gatto, and A. Monaco, "Oropharyngeal airway changes after rapid maxillary expansion: the state of the art," International Journal of Clinical and Experimental Medicine, vol. 7, no. 7, pp. 1632-8, 2014.

[12] E. Ortu, D. Pietropaolio, R. Cattaneo, M. Giannoni, and A. Monaco, "Overjet reduction with the use of the ELIBA device: a case report," Journal of Clinical and Diagnostic Research, vol. 12, no. 12, pp. 8-10, 2018.

[13] F. L. N. Montagna, V. Piras, G. Denotti, and E. I. S. U. O. I. L'ortodonzia, Dispositivi. Apparecchi Mobili E Fissi Rimovibili Nella Pratica Clinica. Orthodontics, fixed and removable devices in clinical practice, 2007. 
[14] S. Tecco, A. Baldini, S. Mummolo et al., "Frenulectomy of the tongue and the influence of rehabilitation exercises on the sEMG activity of masticatory muscles," Journal of Electromyography and Kinesiology, vol. 25, no. 4, pp. 619-628, 2015.

[15] S. F. Dworkin, "Research diagnostic criteria for temporomandibular disorders: current status \& future relevance1," Journal of Oral Rehabilitation, vol. 37, no. 10, pp. 734-743, 2010.

[16] E. Schiffman, R. Ohrbach, E. Truelove et al., "Diagnostic criteria for temporomandibular disorders (DC/TMD) for clinical and research applications: recommendations of the International RDC/TMD Consortium Network and Orofacial Pain Special Interest Group," Journal of Oral \& Facial Pain and Headache, vol. 28, no. 1, pp. 6-27, 2014.

[17] H. M. Michelotti, D. J. de L. Horne, and S. Sheather, "Clinical applications of visual analogue scales: a critical review," Psychological Medicine, vol. 18, no. 4, pp. 1007-1019, 1988.

[18] A. van der Bilt, F. G. Weijnen, F. Bosman, H. W. van der Glas, and J. B. M. Kuks, "Controlled study of EMG activity of the jaw closers and openers during mastication in patients with myasthenia gravis," European Journal of Oral Sciences, vol. 109, no. 3, pp. 160-164, 2001.

[19] T. Castroflorio, D. Farina, A. Bottin, M. G. Piancino, P. Bracco, and R. Merletti, "Surface EMG of jaw elevator muscles: effect of electrode location and inter-electrode distance," Journal of Oral Rehabilitation, vol. 32, no. 6, pp. 411-417, 2005.

[20] H. A. L. Castro, L. A. L. Resende, F. Bérzin, and B. König, "Electromyographic analysis of the superior belly of the omohyoid muscle and anterior belly of the digastric muscle in tongue and head movements," Journal of Electromyography and Kinesiology, vol. 9, no. 3, pp. 229-232, 1999.

[21] D. Falla, P. Dall'Alba, A. Rainoldi, R. Merletti, and G. Jull, "Location of innervation zones of sternocleidomastoid and scalene muscles-a basis for clinical and research electromyography applications," Clinical Neurophysiology, vol. 113, no. 1, pp. 57-63, 2002.

[22] D. Falla, P. Dall'Alba, A. Rainoldi, R. Merletti, and G. Jull, "Repeatability of surface EMG variables in the sternocleidomastoid and anterior scalene muscles," European Journal of Applied Physiology, vol. 87, no. 6, pp. 542-549, 2002.

[23] A. Monaco, R. Cattaneo, L. Mesin, I. Ciarrocchi, F. Sgolastra, and D. Pietropaoli, "Dysregulation of the autonomous nervous system in patients with temporomandibular disorder: a pupillometric study," PLoS One, vol. 7, no. 9, Article ID e45424, 2012.

[24] E. Ortu, D. Pietropaoli, G. Mazzei, R. Cattaneo, M. Giannoni, and A. Monaco, "TENS effects on salivary stress markers: a pilot study," International Journal of Immunopathology and Pharmacology, vol. 28, no. 1, pp. 114-118, 2015.

[25] A. Monaco, R. Cattaneo, E. Ortu, M. V. Constantinescu, and D. Pietropaoli, "Sensory trigeminal ULF-TENS stimulation reduces HRV response to experimentally induced arithmetic stress: a randomized clinical trial," Physiology \& Behavior, vol. 173, pp. 209-215, 2017.

[26] E. Ortu, D. Pietropaoli, F. Adib, C. Masci, M. Giannoni, and A. Monaco, "Electromyographic evaluation in children orthodontically treated for skeletal Class II malocclusion: Comparison of two treatment techniques," Cranio-Journal of Craniomandibular Practice, vol. 37, no. 2, pp. 129-135, 2019.

[27] S. Mummolo, A. Nota, S. Tecco et al., "Ultra-low-frequency transcutaneous electric nerve stimulation (ULF-TENS) in subjects with craniofacial pain: a retrospective study," Cranio, pp. 1-6, 2018.
[28] B. C. Cooper, "The role of bioelectronic instruments in documenting and managing temporomandibular disorders," Journal of the American Dental Association, vol. 127, no. 11, pp. 1611-1614, 1996.

[29] Y. S. Kim, S. Y. Kown, Y. G. Park, and K. R. Chung, "Clinical application of the tongue elevator," Journal of Clinical Orthodontics: JCO, vol. 36, no. 2, pp. 104-6, 2002.

[30] L. Balercia, Elevatore Linguale Balercia, fisiopatologia della deglutizione, Futura Publishing Society, San Benedetto del Tronto, Italy, 1998.

[31] A. Monaco and R. Cattaneo, La TENS per uso odontoiatrico, Futura Publishing Society, San Benedetto del Tronto, Italy, 2016.

[32] A. Monaco and R. Cattaneo, Elettromiografia e chinesiografia per la clinica odontoiatrica, Futura Publishing Society, San Benedetto del Tronto, Italy, 2007 (in Chinese).

[33] A. Monaco, F. Sgolastra, D. Pietropaoli, M. Giannoni, and R. Cattaneo, "Comparison between sensory and motor transcutaneous electrical nervous stimulation on electromyographic and kinesiographic activity of patients with temporomandibular disorder: a controlled clinical trial," $B M C$ Musculoskeletal Disorders, vol. 14, no. 1, p. 168, 2013.

[34] A. Monaco, F. Sgolastra, I. Ciarrocchi, and R. Cattaneo, "Effects of transcutaneous electrical nervous stimulation on electromyographic and kinesiographic activity of patients with temporomandibular disorders: a placebo-controlled study," Journal of Electromyography and Kinesiology, vol. 22, no. 3, pp. 463-468, 2012.

[35] N. Chipaila, F. Sgolastra, A. Spadaro et al., "The effects of ULFTENS stimulation on gnathology: the state of the art," Cranio, vol. 32, no. 2, pp. 118-130, 2014.

[36] T. I. Suvinen and P. Kemppainen, "Review of clinical EMG studies related to muscle and occlusal factors in healthy and TMD subjects," Journal of Oral Rehabilitation, vol. 34, no. 9, pp. 631-644, 2007.

[37] A. Monaco, R. Cattaneo, M. C. Marci, D. Pietropaoli, and E. Ortu, "Central sensitization-based classification for temporomandibular disorders: a pathogenetic hypothesis," Pain Research \& Management, vol. 2017, Article ID 5957076, 13 pages, 2017.

[38] S. Valenzuela, R. Miralles, M. J. Ravera et al., "Does head posture have a significant effect on the hyoid bone position and sternocleidomastoid electromyographic activity in young adults?," Cranio, vol. 23, no. 3, pp. 204-211, 2005.

[39] L. Zheng, J. Jahn, and A. N. Vasavada, "Sagittal plane kinematics of the adult hyoid bone," Journal of Biomechanics, vol. 45, no. 3, pp. 531-536, 2012.

[40] I. J. Edwards, V. K. Lall, J. F. Paton et al., "Neck muscle afferents influence oromotor and cardiorespiratory brainstem neural circuits," Brain Structure and Function, vol. 220, no. 3, pp. 1421-1436, 2015.

[41] H. Santander, R. Miralles, J. Pérez et al., "Effects of head and neck inclination on bilateral sternocleidomastoid EMG activity in healthy subjects and in patients with myogenic cranio-cervical-mandibular dysfunction," Cranio, vol. 18, no. 3, pp. 181-191, 2000.

[42] M. J. Fitzgerald and S. R. Sachithanandan, "The structure and source of lingual proprioceptors in the monkey," Journal of Anatomy, vol. 128, no. Pt 3, pp. 523-52, 1979.

[43] D. Passali, M. C. Spinosi, and F. M. Passali, "Ear nose and throat (ENT) aspects of obstructive sleep apnea syndrome (OSAS) diagnosis and therapy," La Medicina del Lavoro, vol. 108, no. 4, pp. 288-292, 2017.

[44] D. Passali, G. Corallo, A Petti et al., "A comparative study on oxidative stress role in nasal breathing impairment and 
obstructive sleep apnoea syndrome," Acta Otorhinolaryngologica Italica: Organo Ufficiale Della Societa Italiana di Otorinolaringologia e Chirurgia Cervico-Facciale, vol. 36, no. 6, pp. 490-495, 2016.

[45] K. Miyamoto, M. M. Özbek, A. A. Lowe et al., "Mandibular posture during sleep in patients with obstructive sleep apnoea," Archives of Oral Biology, vol. 44, no. 8, pp. 657-664, 1999.

[46] A. J. Miller, "Oral and pharyngeal reflexes in the mammalian nervous system: their diverse range in complexity and the pivotal role of the tongue," Critical Reviews in Oral Biology and Medicine, vol. 13, no. 5, pp. 409-425, 2002.

[47] N. Marchili, E. Ortu, D. Pietropaoli, R. Cattaneo, and A. Monaco, "Dental occlusion and ophthalmology: a literature review," The Open Dentistry Journal, vol. 10, no. 1, pp. $460-468,2016$.

[48] M. Takata, "Lingually induced inhibitory postsynaptic potentials in hypoglossal motoneurons after axotomy," Brain Research, vol. 224, no. 1, pp. 165-169, 1981.

[49] A. A. Lowe, S. C. Gurza, and B. J. Sessle, "Regulation of genioglossus and masseter muscle activity in man," Archives of Oral Biology, vol. 22, no. 10-11, pp. 579-584, 1977.

[50] Y. Ishiwata, T. Ono, T. Kuroda, and Y. Nakamura, "Jawtongue reflex: afferents, central pathways, and synaptic potentials in hypoglossal motoneurons in the cat," Journal of Dental Research, vol. 79, no. 8, pp. 1626-1634, 2000.

[51] P. Bourdiol, A. Mishellany-Dutour, M.-A. Peyron, and A. Woda, "Mood-induced variations of mandible and tongue postures," Journal of Oral Rehabilitation, vol. 40, no. 6, pp. 443-449, 2013.

[52] M. Barnett-Cowan, M. Soeizi, and J. F. X. DeSouza, "Visual attention at the tip of the tongue," i-Perception, vol. 6 , no. 1 , pp. 1-4, 2015.

[53] A. Monaco, V. Cozzolino, R. Cattaneo, T. Cutilli, and A. Spadaro, "Osteopathic manipulative treatment (OMT) effects on mandibular kinetics: kinesiographic study," European Journal of Paediatric Dentistry: Official Journal of European Academy of Paediatric Dentistry, vol. 9, no. 1, pp. 37-42, 2008.

[54] G. D. Klasser and C. S. Greene, "Oral appliances in the management of temporomandibular disorders," Oral Surgery, Oral Medicine, Oral Pathology, Oral Radiology, and Endodontology, vol. 107, no. 2, pp. 212-223, 2009.

[55] Y. Tsukiyama, K. Baba, and G. T. Clark, "An evidence-based assessment of occlusal adjustment as a treatment for temporomandibular disorders," Journal of Prosthetic Dentistry, vol. 86, no. 1, pp. 57-66, 2001.

[56] S. S. Adibi, E. I. Ogbureke, B. B. Minavi, and K. U. Ogbureke, "Why use oral splints for temporomandibular disorders (TMDs)?," Texas Dental Journal, vol. 131, no. 6, pp. 450-455, 2014.

[57] B. C. Cooper, "TMD diagnostics," Journal of the American Dental Association, vol. 144, no. 9, pp. 980-982, 2013. 